\title{
LOCAL UNCERTAINTY INEQUALITIES FOR LOCALLY COMPACT GROUPS
}

\author{
JOHN F. PRICE AND ALLADI SITARAM
}

\begin{abstract}
Let $G$ be a locally compact unimodular group equipped with Haar measure $m, \hat{G}$ its unitary dual and $\mu$ the Plancherel measure (or something closely akin to it) on $\hat{G}$. When $G$ is a euclidean motion group, a noncompact semisimple Lie group or one of the Heisenberg groups we prove local uncertainty inequalities of the following type: given $\theta \in\left[0, \frac{1}{2}\right)$ there exists a constant $K_{\theta}$ such that for all $f$ in a certain class of functions on $G$ and all measurable $E \subseteq \hat{G}$,
\end{abstract}

$$
\left(\int_{E} \operatorname{Tr}\left(\pi(f)^{*} \pi(f)\right) d \mu(\pi)\right)^{1 / 2} \leq K_{\theta} \mu(E)^{\theta}\left\|\phi_{\theta} f\right\|_{2}
$$

where $\phi_{\theta}$ is a certain weight function on $G$ (for which an explicit formula is given). When $G=\mathbf{R}^{k}$ the inequality has been established with $\phi_{\theta}(x)=|x|^{k \theta}$.

1. Introduction. Throughout $G$ denotes a locally compact group equipped with left Haar measure $d m$. (Instead of $\int_{G} f(x) d m(x)$ we will sometimes write $\int_{G} f(x) d x$ or $\int_{G} f d m$.) Denote the dual of $G$ by $\hat{G}$, that is, $\hat{G}$ is a maximal set of pairwise inequivalent unitary irreducible (continuous) representations of $G$. For each $\pi \in \hat{G}, H_{\pi}$ will denote the corresponding Hilbert space. The Fourier transform $\hat{f}$ of $f \in L^{1}(G)$ is defined by $\hat{f}(\pi)=\pi(f)=\int_{G} f(x) \pi(x) d x$ for $\pi \in \hat{G}$. Hence $\pi(f) \in B\left(H_{\pi}\right)$, the space of bounded linear operators on $H_{\pi}$.

Recently we showed for certain groups $G$ (including the motion group, the affine group, the Heisenberg group and all semisimple Lie groups (under some extra conditions)) that if $f \in L^{1}(G) \cap L^{2}(G)$ satisfies $m\{x \in G: f(x) \neq 0\}<\infty$ and $\mu\{\pi: \pi(f) \neq 0\}<\infty$ then $f=0$ a.e. [11]. (Here $\mu$ denotes some type of "measure" on $\hat{G}$ closely related to the Plancherel measure. Also in some cases the restriction on $f$ may be more stringent, in others more relaxed.) Benedicks [1] established this result for $G=\mathbf{R}^{k}$ with $m$ and $\mu$ denoting Lebesgue measure.

This result is a simple type of uncertainty principle since it places a restriction on the amount to which both a function and its Fourier transform can be concentrated. In the following we make this idea more precise for certain groups by establishing local uncertainty inequalities.

In euclidean Fourier analysis uncertainty principles state that the more a function is concentrated about some point, the more its Fourier transform must be spread, and vice versa. However, they do not preclude that this spreading (which is usually measured as a standard deviation) may be achieved by two or more peaks far apart.

Received by the editors November 21, 1986.

1980 Mathematics Subject Classification (1985 Revision). Primary 43A30, 22E30; Secondary $43 \mathrm{~A} 80$. 
Local uncertainty principles state that if a function is concentrated, then not only is its Fourier transform spread out, but that it cannot be "too localized" at any point. A variety of inequalities supporting these latter principles can be found in $[\mathbf{3}, \mathbf{9}, \mathbf{1 0}]$ along with applications.

Of central interest to this paper is the following slight sharpening of a local uncertainty inequality proved in [9]. Because its proof provides a blueprint for the main results below, Theorem 3.1 and its Corollary, we give a sketch of it here. Most of the steps are made up of familiar inequalities but for more details see [9]. We also indicate in the proofs of two corollaries how local uncertainty inequalities imply global uncertainty inequalities, in particular, the classical uncertainty inequality involving standard deviations. The notation used in the remainder of this section is as follows: Given $f \in L^{1}\left(\mathbf{R}^{k}\right)$, its euclidean Fourier transform $\hat{f}$ is defined by

$$
\hat{f}(y)=\int f(x) e^{-2 \pi i x y} d x
$$

where $x y=x_{1} y_{1}+\cdots+x_{k} y_{k}$ and, unless stated otherwise, $\int \ldots d x$ denotes integration over $\mathbf{R}^{k}$ with respect to Lebesgue measure. Let $\omega_{k}=2 \pi^{k / 2} / \Gamma(k / 2)$; when $k \in \mathbf{Z}^{+}=\{1,2, \ldots\}$ and $k>1$, this is the surface area of the unit ball in $\mathbf{R}^{k}$. Generally $x$ will be the variable used with $f$ and $y$ with its Fourier transform $\hat{f}$.

1.1 TheOREM. Suppose $0 \leq \theta<1 / 2$. For all measurable sets $E \subseteq R^{k}$ with Lebesgue measure $m(E)<\infty$ and all functions $f \in L^{2}\left(\mathbf{R}^{k}\right)$,

$$
\left(\int_{E}|\hat{f}(y)|^{2} d y\right)^{1 / 2} \leq K_{1} m(E)^{\theta}\left\||x|^{k \theta} f\right\|_{2}
$$

where

$$
K_{1}=\left(\omega_{k} /(2 \theta)^{2} k\right)^{\theta}(1-2 \theta)^{\theta-1} .
$$

Furthermore, no inequality of this form is possible (a) for any other power of $m(E)$, and (b) for $\theta$ outside $\left[0, \frac{1}{2}\right)$.

ProOF. Let $B$ denote the closed unit ball in $\mathbf{R}^{k}$ and $B^{\prime}$ its complement. Denote by $1_{r}$ and $1_{r^{\prime}}$ the characteristic functions $1_{r B}$ and $1_{r B^{\prime}}$ respectively. Given $f, E$ and $\theta$ as in the statement of the theorem,

$$
\begin{aligned}
\left(\int_{E}|\hat{f}|^{2}\right)^{1 / 2} & \leq\left\|\left(f 1_{r}\right)^{\wedge} 1_{E}\right\|_{2}+\left\|\left(f 1_{r^{\prime}}\right)^{\wedge} 1_{E}\right\|_{2} \quad \text { where } r>0 \\
& \leq m(E)^{1 / 2}\left\|\left(f 1_{r}\right)^{\wedge}\right\|_{\infty}+\left\|f 1_{r^{\prime}}\right\|_{2} \\
& \leq m(E)^{1 / 2}\left\|f 1_{r}\right\|_{1}+\left\|1_{r^{\prime}}|x|^{-k \theta}\right\|_{\infty}\left\|\left.x\right|^{k \theta} f\right\|_{2} \\
& \leq m(E)^{1 / 2}\left\|1_{r}|x|^{-k \theta}\right\|_{2}\left\|1_{r}|x|^{k \theta} f\right\|_{2}+r^{-k \theta}\left\||x|^{k \theta} f\right\|_{2} \\
& \leq\left(m(E)^{1 / 2}\left(\frac{\omega_{k}}{k(1-2 \theta)}\right)^{1 / 2} r^{k(1-2 \theta) / 2}+r^{-k \theta}\right)\left\||x|^{k \theta} f\right\|_{2} .
\end{aligned}
$$

By choosing $r>0$ to satisfy

$$
r^{k}=(2 \theta k)^{2} / m(E) \omega_{k} k(1-2 \theta)
$$

we minimize the right side to obtain the desired inequality.

Proofs of the facts contained in the last sentence of the statement of the theorem are given in [9]. 
1.2 Corollary. Suppose $0 \leq \alpha<k / 2$. For all functions $f \in L^{2}\left(\mathbf{R}^{k}\right)$

$$
\|f\|_{2}^{2} \leq K_{2}\left\||x|^{\alpha} f\right\|_{2}\left\||y|^{\alpha} \hat{f}\right\|_{2}
$$

where

$$
K_{2}=2 \frac{\omega_{k}^{2 \alpha / k}}{(2 \alpha k)^{\alpha k}(1-2 \alpha / k)^{1 / 2}} .
$$

Proof. Take $E=\left\{y \in \mathbf{R}^{k}:|y| \leq r\right\}$ in the statement of Theorem 1.1; then $m(E)=r^{k} \omega_{k} / k$. Let $\theta=\alpha / k$ and $E^{\prime}$ be the complement of $E$. Then

$$
\begin{aligned}
\|f\|_{2}^{2} & =\|\hat{f}\|_{2}^{2}=\int_{E}|\hat{f}|^{2}+\int_{E^{\prime}}|\hat{f}|^{2} \\
& \leq K_{1}^{2}\left(\omega_{k} / k\right)^{2 \alpha / k} r^{2 \alpha}\left\||x|^{\alpha} f\right\|_{2}^{2}+r^{-2 \alpha}\left\||y|^{\alpha} \hat{f}\right\|_{2}^{2} .
\end{aligned}
$$

Now minimize the right side by taking

$$
r=\left(\frac{\left\||y|^{\alpha} \hat{f}\right\|_{2}}{K_{1}\left\||x|^{\alpha} f\right\|_{2}}\right)^{1 / 2 \alpha}\left(\frac{\omega_{k}}{k}\right)^{-1 / 2 k}
$$

to get the required inequality.

The inequality $\left\||x|^{\alpha} f\right\|_{2} \leq\|f\|_{2}^{1-\alpha / \beta}\left\||x|^{\beta} f\right\|_{2}^{\alpha / \beta}$ for $0<\alpha<\beta$ applied to $f$ and $\hat{f}$ allows the following to be deduced from Corollary 1.2 .

1.3 COROLlary. Given $\beta \geq 0$, there exists a constant $K$ such that

$$
\|f\|_{2}^{2} \leq K\left\||x|^{\beta} f\right\|_{2}\left\||y|^{\beta} \hat{f}\right\|_{2}
$$

for all $f \in L^{2}\left(\mathbf{R}^{k}\right)$.

Inequalities of the form described in Corollaries 1.2 and 1.3 are examined in detail in [2]. When $\beta=1$ the inequality has the same general form as the classical Heisenberg-Pauli-Weyl inequality [2]. See also [7].

In this paper we prove analogues of Theorem 1.1 for semisimple Lie groups with finite centres, motion groups and the Heisenberg groups. In the case of symmetric spaces we deduce a global uncertainty principle from the local one in the same manner as Corollaries 1.2 and 1.3 were deduced from Theorem 1.1.

The second-named author thanks the University of New South Wales for its hospitality during the preparation of this paper. This visit was supported by the Australian Research Grants Scheme. We are also both grateful to Michael Cowling for numerous helpful conversations.

2. Semisimple Lie groups and motion groups. In this section we introduce some notation and quote some results from Warner [14] which will be used in the sequel. In $\S \S 3$ and 4 we will be concerned with the following two classes of groups G: (a) Noncompact, connected semisimple Lie groups with finite centre and (b) euclidean motion groups, that is, semidirect products of compact groups $K$ and $\mathbf{R}^{n}$, where $K$ acts as a group of linear automorphisms of $\mathbf{R}^{n}$. In the case of (a) above, $K$ will always denote a fixed maximal compact subgroup of $G$. Thus in both cases $G$ can be written as $G=\mathbf{R}^{n} \cdot K$ and topologically $G$ is homeomorphic to $\mathbf{R}^{n} \times K$. Always $\hat{G}$ will denote the dual of $G$. For the groups above, which are all unimodular, one has the Plancherel theorem due to I. Segal (see [14, vol. II, p. $52])$. 
2.1 THEOREM. Fix a Haar measure $d m$ on a Lie group $G$, where $G$ belongs to one of the classes just described. Then there exists a unique positive measure $\mu$ on $\hat{G}$ such that

$$
\int_{G}|f(x)|^{2} d m(x)=\int_{\hat{G}} \operatorname{Tr}\left(\pi(f)^{*} \pi(f)\right) d \mu(\pi)
$$

for all $f \in L^{1}(G) \cap L^{2}(G)$.

2.2 REMARKS. For any $\pi \in \hat{G}$ and $f \in L^{1}(G)$ the operator $\pi(f)$ is well defined and acts on $H_{\pi}$, the underlying Hilbert space of $\pi$. The assignment $\pi \mapsto \hat{f}(\pi)=$ $\pi(f)$ is the group theoretic analogue of the usual Fourier transform and given $E \subseteq$ $\hat{G}, \int_{E} \operatorname{Tr}\left(\pi(f)^{*} \pi(f)\right) d \mu(\pi)$ measures the 'amount' of the Fourier transform that 'lives' on $E$. The measure $\mu$ is called the Plancherel measure for $\hat{G}$ (associated with the given Haar measure on $G$ ). If the Haar measure is multiplied by a positive scalar $k$, then the Plancherel measure is multiplied by $k^{-1}$. Implicit in the statement of the above theorem is the fact that $\pi(f)$ (for $f \in L^{1}(G) \cap L^{2}(G)$ ) is of Hilbert-Schmidt class for $\mu$-almost all $\pi(\pi \in \hat{G})$.

Let $\hat{K}$ denote the dual of the compact group $K$. (Here $G, K$ are as in (a) or (b) in the first paragraph.) Then by a theorem of Harish-Chandra for semisimple $G$ and a theorem of Godement for general motion groups [14, vol. I, p. 314 and p. 319] one has:

2.3 THEOREM. A given $\delta \in \hat{K}$ occurs no more than $d(\delta)$ times in the restriction to $K$ of any irreducible unitary representation $\pi$ of $G$. (Here $d(\delta)$ denotes the dimension of $\delta$.)

Next we introduce a class of right $K$-finite functions on $G$. For $\delta \in \hat{K}$, let $R_{\delta}$ be the linear subspace of $C(K)$ consisting of representative functions of type $\delta$, that is, the linear span of the matrix elements corresponding to $\delta$. Let $F$ be a finite subset of $\hat{K}$. We say a function $f \in L^{p}(G)$ is right $K$-finite of type $F$ (or simply of type $F$ ) if there exist finitely many functions $\phi_{1}, \ldots, \phi_{l}$ in $L^{p}(G)$ and $a_{1}, \ldots, a_{l}$ in $\bigoplus \sum_{\delta \in F} R_{\delta^{*}}$ (orthogonal direct sum) such that $f^{k}=a_{1}(k) \phi_{1}+\cdots+a_{l}(k) \phi_{l}$, for all $k \in K$. Here $f^{k}$ denotes the right translate of $f$ by the element $k$, that is, $f^{k}(x)=f(x k), x \in G$, and $\delta^{*}$ denotes the representation contragredient to $\delta$. Denote by $L_{F}^{p}(G)$ the subspace of $L^{p}(G)$ consisting of functions of type $F$. For use in $\S 3$ we record the following two lemmas - the proofs are quite easy and so we omit them.

2.4 LEMMA. If $g$ is a bounded measurable function which is right $K$-invariant and $f \in L_{F}^{p}(G)$, then $f g \in L_{F}^{p}(G)$.

2.5 LEMMA. Let $(\pi, H)$ be an irreducible unitary representation of $G$. For each $\delta \in \hat{K}$, let $H_{\delta}$ be the linear subspace of $H$ of vectors transforming according to $\delta$ for the representation $\left(\left.\pi\right|_{K}, H\right)$ of $K$. [Then one knows that $\operatorname{dim} H_{\delta} \leq d(\delta)^{2}$ (see Theorem 2.3) and $H=\bigoplus \sum_{\delta \in \hat{K}} H_{\delta}$.] If $f \in L_{F}^{1}(G)$ then $\pi(f)$ is zero on $\left(\sum_{\delta \in F} H_{\delta}\right)^{\perp}$. 
Let $N(F)=\sum_{\delta \in F} d(\delta)^{2}$. By Theorem 2.3 one knows that $\operatorname{dim}\left(\bigoplus \sum_{\delta \in F} H_{\delta}\right) \leq$ $N(F)$. Combining this with the above lemma one has for $f \in L_{F}^{1}(G)$ :

$$
\operatorname{Tr}\left(\pi(f)^{*} \pi(f)\right)=\sum_{i=1}^{N}\left\langle\pi(f)^{*} \pi(f) e_{i}, e_{i}\right\rangle=\sum_{i=1}^{N}\left\langle\pi(f) e_{i}, \pi(f) e_{i}\right\rangle
$$

where $e_{1}, \ldots, e_{N}$ is an orthonormal basis for $\bigoplus \sum_{\delta \in F} H_{\delta}$. As observed above $N \leq$ $N(F)$ and so we easily get the following.

\subsection{LEMMA. For $f \in L_{F}^{1}(G)$ and $\pi \in \hat{G}, \operatorname{Tr}\left(\pi(f)^{*} \pi(f)\right) \leq N(F)\|f\|_{1}^{2}$.}

We will now introduce the notion of a "norm" on $G$ which in some sense will tell us how far an element is from $e$ or more precisely from $K$, the maximal compact subgroup.

Case 1. $G$ semisimple. For what follows a good reference is [6]. Let $\mathfrak{g}$ be the Lie algebra of $G$ and $\mathfrak{g}=\mathfrak{k} \oplus \mathfrak{p}$ the Cartan decomposition of $\mathfrak{g}(\mathfrak{k}$ is the Lie algebra of $K)$. Then one knows that the Killing form $B$ restricted to $\mathfrak{p}$ is positive definite. Let $P=\exp \mathfrak{p}$. Then $P$ is diffeomorphic to $\mathfrak{p}$ under the exponential map. Also $G=P K$ and $G$ is diffeomorphic to $P \times K$ under $(u, k) \mapsto u k, u \in P, k \in K$. So each $g \in G$ can be uniquely written as $g=g_{P} g_{K}$ with $g_{P} \in P$ and $g_{K} \in K$. From what we said above $g_{P}=\exp X$ for a unique element $X \in \mathfrak{p}$. Define $\|g\|=(B(X, X))^{1 / 2}$. Then $\|\cdot\|$ gives a continuous (in fact smooth) map from $G$ into $R^{+} \cup\{0\}$ with the property that $\|g\| \rightarrow \infty$ if $g \rightarrow \infty$ in $G$ and $\|g\| \rightarrow 0$ if $g \rightarrow e$ (or even if " $g \rightarrow K$ ") in $G$.

Case 2. $G$ a euclidean motion group. In this case $G=\mathbf{R}^{n} \cdot K$ and is homeomorphic to $\mathbf{R}^{n} \times K$. Every $g \in G$ can be uniquely written as $g=g_{t} g_{K}$ where $g_{t} \in \mathbf{R}^{n}$ and $g_{K} \in K$. Define $\|g\|=\left\|g_{t}\right\|=$ euclidean norm of $g_{t}$. Here again $\|\cdot\|$ is a continuous map from $G$ into $\mathbf{R}^{+} \cup\{0\}$ and $\|g\| \rightarrow \infty$ if $g \rightarrow \infty$ in $G$ and $\|g\| \rightarrow 0$ if $g \rightarrow e$ (or even if " $g \rightarrow K$ ") in $G$.

Finally we will introduce a function which will help us measure the concentration of a function around $K$. Let $A$ be the function on $\mathbf{R}^{+} \cup\{0\}$ defined by: $A(r)=$ $m_{G}\{g \in G:\|g\| \leq r\}$. Here $m_{G}$ denotes Haar measure on $G$. Then $A$ is a continuous strictly increasing function of $r$ and $A(r) \rightarrow 0+$ as $r \rightarrow 0+$ and $A(r) \rightarrow \infty$ as $r \rightarrow \infty$. Note that $A(0)=m_{G}(K)=0$.

2.7 REMARK. In the case when $G$ is semisimple, consider the symmetric space $X=G / K$ equipped with its canonical riemannian structure and the canonical $G$ invariant riemannian measure. Then $A(r)$ is just the volume of the geodesic ball in $X$ of radius $r$ around $e K$.

3. Local uncertainty inequalities. The purpose of this section is to prove a local uncertainty principle for $K$-finite functions on $G$ which is analogous to the local uncertainty principle for functions on $\mathbf{R}^{n}$ proved in $\S 1$. Roughly speaking it says that the more concentrated a $K$-finite function is around $K$, the more spread out is its Fourier transform on $\hat{G}$. Retaining the notation of the previous section we now state the main result of this section: 
3.1 THEOREM. Let $F$ be a finite subset of $\hat{K}$ and $0<\theta<\frac{1}{2}$. Then there exists a positive constant $C_{F, \theta}$ such that

$$
\left(\int_{E} \operatorname{Tr}\left(\pi(f)^{*} \pi(f)\right) d \mu(\pi)\right)^{1 / 2} \leq C_{F, \theta} \mu(E)^{\theta}\left\|[A(\|\cdot\|)]^{\theta} f\right\|_{2}
$$

for all $f \in L_{F}^{1}(G) \cap L_{F}^{2}(G)$ and measurable $E \subseteq \hat{G}$.

ProOF. Let $B_{a}=\{g \in G:\|g\| \leq a\}$ and $B_{a}^{\prime}$ its complement. Then $f=f 1_{B_{a}}+$ $f 1_{B_{a}^{\prime}}$. Let $f 1_{B_{a}}=f_{1}$ and $f 1_{B_{a}^{\prime}}=f_{2}$. Both $1_{B_{a}}$ and $1_{B_{a}^{\prime}}$ are right $K$-invariant functions (because $x \in B_{a}$ if and only if $x k \in B_{a}, k \in K$ ). Hence by Lemma $2.4, f_{1}$ and $f_{2}$ are also in $L_{F}^{1}(G) \cap L_{F}^{2}(G)$. Since $g \mapsto\left(\int_{E} \operatorname{Tr}\left(\pi(f)^{*} \pi(f)\right) d \mu(\pi)\right)^{1 / 2}$ satisfies the triangle inequality we have

$$
\begin{aligned}
& \left(\int_{E} \operatorname{Tr}\left(\pi(f)^{*} \pi(f)\right) d \mu(\pi)\right)^{1 / 2} \\
& \quad \leq\left(\int_{E} \operatorname{Tr}\left(\pi\left(f_{1}\right)^{*} \pi\left(f_{1}\right)\right) d \mu(\pi)\right)^{1 / 2}+\left(\int_{E} \operatorname{Tr}\left(\pi\left(f_{2}\right)^{*} \pi\left(f_{2}\right)\right) d \mu(\pi)\right)^{1 / 2} .
\end{aligned}
$$

Now by Lemma 2.6

$$
\operatorname{Tr}\left(\pi\left(f_{1}\right)^{*} \pi\left(f_{1}\right)\right) \leq N(F)\left\|f_{1}\right\|_{1}^{2} .
$$

Hence the first term on the right side of $(1)$ is dominated by $\mu(E)^{1 / 2} N(F)^{1 / 2}\left\|f_{1}\right\|_{1}$. By the Plancherel theorem the second term on the right side of $(1)$ is dominated by $\left\|f_{2}\right\|_{2}=\left\|f 1_{B_{a}^{\prime}}\right\|_{2}$. Hence

$$
\left(\int_{E} \operatorname{Tr}\left(\pi(f)^{*} \pi(f)\right) d \mu(\pi)\right)^{1 / 2} \leq N(F)^{1 / 2} \mu(E)^{1 / 2}\left\|f 1_{B_{a}}\right\|_{1}+\left\|f 1_{B_{a}^{\prime}}\right\|_{2} .
$$

Exactly as in the euclidean case, the first term on the right side of (2) is dominated by

$$
\mu(E)^{1 / 2} N(F)^{1 / 2}\left\|[A(\|\cdot\|)]^{-\theta} 1_{B_{a}}\right\|_{2}\left\|[A(\|\cdot\|)]^{\theta} f\right\|_{2}
$$

(by Cauchy-Schwarz). The second term on the right side of (2) is clearly dominated by $A(a)^{-\theta}\left\|[A(\|\cdot\|)]^{\theta} f\right\|_{2}$ (because $A$ is an increasing function). Thus:

$$
\begin{aligned}
\left(\int _ { E } \operatorname { T r } \left(\pi(f)^{*} \pi\right.\right. & (f)) d \mu(\pi))^{1 / 2} \\
\leq & m(E)^{1 / 2} N(F)^{1 / 2}\left\|A(\|\cdot\|)^{-\theta} 1_{B_{a}}\right\|_{2}\left\|A(\|\cdot\|)^{\theta} f\right\|_{2} \\
& +A(a)^{-\theta}\left\|A(\|\cdot\|)^{\theta} f\right\|_{2} .
\end{aligned}
$$

Now

$$
\left\|A(\|\cdot\|)^{-\theta} 1_{B_{a}}\right\|_{2}=\left(\int_{0}^{a} A(t)^{-2 \theta} d A(t)\right)^{1 / 2}=\left|A(a)^{1-2 \theta} /(1-2 \theta)\right|^{1 / 2} .
$$

Using this, the right side of $(3)$ becomes

$$
\left[\mu(E)^{1 / 2} N(F)^{1 / 2}\left|A(a)^{1-2 \theta} /(1-2 \theta)\right|^{1 / 2}+A(a)^{-\theta}\right]\left\|A(\|\cdot\|)^{\theta} f\right\|_{2} .
$$


Now, using the facts that $A(0)=m(K)=0$ and that $A(t)$ takes all values between 0 and $\infty$, arguing exactly as in the euclidean case (that is, choose $a$ such that $A(a)=$ constant $/ \mu(E))$ we finally have

$$
\left(\int_{E} \operatorname{Tr}\left(\pi(f)^{*} \pi(f)\right) d \mu(\pi)\right)^{1 / 2} \leq C_{F, \theta} \mu(E)^{\theta}\left\|[A(\|\cdot\|)]^{\theta} f\right\|_{2} \text {. Q.E.D. }
$$

We can restate Theorem 3.1 in a form that is slightly closer to the classical local uncertainty principle discussed in $\S 1$. Before that we introduce some notation. For $\delta \in \hat{K}$ define $\chi_{\delta}(k)=d(\delta) \operatorname{Tr}(\delta(k))$ for $k \in K$. For a function $f$ on $G$ define $f_{\delta}$ by

$$
f_{\delta}(x)=\left(f * \chi_{\delta}\right)(x)=\int_{K} f(x k) \chi_{\delta}\left(k^{-1}\right) d k .
$$

Then if $f \in L^{p}(G), f_{\delta}$ is right $K$-finite of type $F$ where $F=\{\delta\}$. Thus Theorem 3.1 is applicable to $f_{\delta}$ and in fact we can prove:

3.2 COROLlaRY. For each $\delta \in \hat{K}$ and $0<\theta<\frac{1}{2}$, there exists a positive constant $C_{\theta, \delta}$ such that

$$
\left(\int_{E} \operatorname{Tr}\left(\pi\left(f_{\delta}\right)^{*} \pi\left(f_{\delta}\right)\right) d \mu(\pi)\right)^{1 / 2} \leq C_{\theta, \delta} \mu(E)^{\theta}\left\|A(\|\cdot\|)^{\theta} f\right\|_{2}
$$

for all $f$ in $L^{1}(G) \cap L^{2}(G)$ and measurable $E \subseteq \hat{G}$.

PrOOF. From the discussion above and Theorem 3.1 we have

$$
\left(\int_{E} \operatorname{Tr}\left(\pi\left(f_{\delta}\right)^{*} \pi\left(f_{\delta}\right)\right) d \mu(\pi)\right)^{1 / 2} \leq C_{\theta, \delta} \mu(E)^{\theta}\left\|A(\|\cdot\|)^{\theta} f_{\delta}\right\|_{2} .
$$

Now observe that if $g$ is a right $K$-invariant function, then $(g f)_{\delta}=g f_{\delta}$. Also for any $h,\left\|h_{\delta}\right\|_{2} \leq\|h\|_{2}$ because $h=\bigoplus \sum_{\gamma \in \hat{K}} h_{\gamma}$ is an orthogonal decomposition. The proof of the corollary is complete once we combine these observations with the fact that the function $A(\|\cdot\|)^{\theta}$ is a right $K$-invariant function on $G$.

4. A global uncertainty inequality for symmetric spaces. In this section we will indicate how the local uncertainty principle for the semisimple Lie group $G$ leads to a global uncertainty principle-at least for the symmetric space $G / K$. Since the details are very similar to the euclidean case, we refer the reader to $\S 1$ on how the local uncertainty principle implies the global one.

Consider $X=G / K$, a riemannian symmetric space of the noncompact type, equipped with its canonical riemannian structure. Here $G$ is a noncompact connected semisimple Lie group with finite centre, $K$ a fixed maximal compact subgroup and $G$ can be identified with the connected component of the group of isometries of $X$. Let $G=K A N$ be an Iwasawa decomposition of $G$ and $\mathfrak{a}$ the Lie algebra of $A$ and $\mathfrak{a}^{*}$ its dual. Let $\|x\|$ denote the distance of $x \in X$ to $e K \in X$ (in the riemannian metric). Let $m$ be the canonical $G$-invariant measure on $X$ induced by the riemannian structure. In fact the Haar measure $d g$ on $G$ can be so normalized that $\int_{G} f(g) d g=\int_{X} f(x) d m(x)$ for right $K$-invariant functions on $G$. (On the right side above we are interpreting a right $K$-invariant function as a function on $X$ via the identification $f(g K)=f(g)$.) As before, let $A(r)=m\{x:\|x\| \leq r\}$. Let $\left\{\pi_{\lambda}\right\}_{\lambda \in a^{*}}$ be the class-1 (that is, spherical) principal-series representations of $G$ (see [5]) and 
let $\mu$ be the (Harish-Chandra) Plancherel measure on $\mathfrak{a}^{*}$ normalized so that one has the equality

$$
\int_{X}|f(x)|^{2} d m(x)=\int_{\mathfrak{a}^{*}} \operatorname{Tr}\left(\pi_{\lambda}(f)^{*} \pi_{\lambda}(f)\right) d \mu(\lambda)
$$

for all $f \in L^{1}(X) \cap L^{2}(X)\left(=L^{1} \cap L^{2}(G / K)\right)$. (Let $W$ be the Weyl group of the pair $(G, A)$; then $\pi_{\lambda}$ will be equivalent to $\pi_{s \lambda}$ for $s \in W$ and these are the only possible identifications. Thus $\mu$ will be a $W$-invariant measure.) Then the local uncertainty principle of the previous section can be reformulated as:

Given $0<\theta<\frac{1}{2}$, there exists a constant $C_{\theta}$ such that for all $f \in L^{1}(X) \cap L^{2}(X)$ and measurable $E \subseteq \mathfrak{a}^{*}$, we have

$$
\left[\int_{E} \operatorname{Tr}\left(\pi_{\lambda}(f)^{*} \pi_{\lambda}(f)\right) d \mu(\lambda)\right]^{1 / 2} \leq C_{\theta} \mu(E)^{\theta}\left(\int_{X} A(\|x\|)^{2 \theta}|f(x)|^{2} d m(x)\right)^{1 / 2} .
$$

Now let $B$ be the function on $\mathbf{R}^{+} \cup\{0\}$ defined by $B(t)=\mu\left\{\lambda \in \mathfrak{a}^{*}:\|\lambda\|_{\mathfrak{a}^{*}} \leq t\right\}$. Here $\|\cdot\|_{\mathfrak{a}^{*}}$ denotes the norm on $\mathfrak{a}^{*}$ induced by the Killing form restricted to $\mathfrak{a}$. Then arguing exactly as in the euclidean case one gets a global uncertainty principle.

Given $0<\theta<\frac{1}{2}$, there exists a constant $K_{\theta}$ such that for all $f \in L^{1}(X) \cap L^{2}(X)$ we have

$$
\left(\int_{X} A(\|x\|)^{2 \theta}|f(x)|^{2} d m(x)\right)\left(\int_{\mathfrak{a}^{*}} B\left(\|\lambda\|_{\mathfrak{a}^{*}}\right)^{2 \theta} \operatorname{Tr}\left(\pi_{\lambda}(f)^{*} \pi_{\lambda}(f)\right) d \mu(\lambda)\right) \geq K_{\theta}\|f\|_{2}^{4} .
$$

Again from this one can get the usual version of the uncertainty principle exactly as in the euclidean case, that is, there exists a constant $K$ such that for all $f \in$ $L^{1} \cap L^{2}(X)$ one has

$$
\left(\int_{X} A(\|x\|)^{2}|f(x)|^{2} d m(x)\right)\left(\int_{\mathfrak{a}^{*}} B\left(\|\lambda\|_{\mathfrak{a}^{*}}\right)^{2} \operatorname{Tr}\left(\pi_{\lambda}(f)^{*} \pi_{\lambda}(f)\right) d \mu(\lambda)\right) \geq K\|f\|_{2}^{4} .
$$

REMARK. The quantity $\operatorname{Tr}\left(\pi_{\lambda}(f)^{*} \pi_{\lambda}(f)\right)$ is just $\left(f^{*} * f\right)^{-}(\lambda)$ where - denotes the spherical Fourier transform-see [5]. In particular, if $f$ is $K$-biinvariant $\left(f^{*} * f\right)^{\wedge}(\lambda)=|\hat{f}(\lambda)|^{2}$ and the last inequality becomes

$$
\left(\int_{X} A(\|x\|)^{2}|f(x)|^{2} d m(x)\right)\left(\int_{\mathfrak{a}^{*}} B\left(\|\lambda\|_{\mathfrak{a}^{*}}\right)^{2}|\hat{f}(\lambda)|^{2} d \mu(\lambda)\right) \geq K\|f\|_{2}^{4},
$$

which reads exactly like the classical Heisenberg uncertainty principle.

5. The Heisenberg groups $H_{n}$. The Heisenberg group $\mathbf{H}_{n}$ is just $\mathbf{R}^{2 n+1}$ with multiplication defined as follows:

$$
(p, q, t)\left(p^{\prime}, q^{\prime}, t^{\prime}\right)=\left(p+p^{\prime}, q+q^{\prime}, t+t^{\prime}+\frac{1}{2}\left(p \cdot q^{\prime}-p^{\prime} \cdot q\right)\right)
$$

where $p, p^{\prime}, q, q^{\prime} \in \mathbf{R}^{n}, t, t^{\prime} \in \mathbf{R}$ and $\cdot$ denotes the usual inner product in $\mathbf{R}^{n} . \mathbf{H}_{n}$ is a simply connected (two step) nilpotent Lie group. Its Haar measure is just $d p d q d t$. For each $\lambda \in \mathbf{R}^{\times}=\mathbf{R} \backslash\{0\}$, one can define an irreducible unitary representation $\pi_{\lambda}$ on $L^{2}\left(\mathbf{R}^{n}\right)$ by

$$
\left(\pi_{\lambda}(p, q, t) f\right)(x)=e^{2 \pi i q \cdot x+\pi i \lambda p \cdot q+2 \pi i h t} f(x+\lambda p) .
$$

$\left\{\pi_{\lambda}\right\}_{\lambda \in \mathbf{R} \backslash\{0\}}$ is a family of inequivalent irreducible representations and in fact the Plancherel measure is concentrated on this family: it is given by $d \mu=|\lambda|^{n} d \lambda$. 
Denote the set of these representations by $\hat{G}_{r}\left(\leftrightarrow \mathbf{R}^{\times}\right)$. Thus the Plancherel theorem for $\mathbf{H}_{n}$ takes the following concrete form: For $f \in L^{1}\left(\mathbf{H}_{n}\right) \cap L^{2}\left(\mathbf{H}_{n}\right)$,

$$
\int \operatorname{Tr}\left(\pi_{\lambda}(f)^{*} \pi_{\lambda}(f)\right)|\lambda|^{n} d \lambda=\|f\|_{2}^{2}
$$

All this is essentially given by the Stone-von Neumann theorem. For the case $n=1$, a discussion can be found in [13]-however the parametrization of the group as well as the dual is slightly different from ours.

For a function $f$ on $\mathbf{H}_{n}\left(=\mathbf{R}^{2 n+1}\right)$ which is sufficiently nice, for example $f \in$ $C_{c}^{\infty}\left(\mathbf{H}_{n}\right)$, one can easily show from the above that the Fourier transform $\pi_{\lambda}(f)$ of $f$ at $\lambda \in \mathbf{R}^{\times}$is given by the following operator on $L^{2}\left(\mathbf{R}^{n}\right)$ :

$$
\left(\pi_{\lambda}(f) \phi\right)(x)=\int k_{\lambda}(x, w) \phi(w) d w
$$

where

$$
k_{\lambda}(x, w)=\frac{1}{|\lambda|^{n}}\left(F_{23} f\right)\left(\frac{w-x}{\lambda}, \frac{w+x}{2}, \lambda\right) .
$$

Here $F_{23} f$ is the ordinary (euclidean) Fourier transform of $f$ in the second and third (families of) variables. Thus $\pi_{\lambda}(f)$ is an integral operator on $L^{2}\left(\mathbf{R}^{n}\right)$ with kernel $K_{\lambda}$. It will therefore follow from standard facts about integral operators that

$$
\operatorname{Tr}\left(\pi_{\lambda}(f)^{*} \pi_{\lambda}(f)\right)=\iint\left|K_{\lambda}(x, w)\right|^{2} d x d w
$$

Thus

$$
\operatorname{Tr}\left(\pi_{\lambda}(f)^{*} \pi_{\lambda}(f)\right)=\iint \frac{1}{|\lambda|^{2 n}}\left|F_{23} f\left(\frac{w-x}{\lambda}, \frac{w+x}{2}, \lambda\right)\right|^{2} d x d w
$$

which after an obvious change of variables reduces to

$$
\iint \frac{1}{|\lambda|^{2 n}}\left|F_{23} f(u, v, \lambda)\right|^{2}|\lambda|^{n} d u d v
$$

The following is the main result for this section. (In a series of papers, Schempp has looked at the radar uncertainty principle for the Heisenberg groups. See, for example, [12].)

5.1 ThEOREM. Given $\theta \in\left[0, \frac{1}{2}\right)$, for each $f \in L^{1}\left(\mathbf{H}_{n}\right) \cap L^{2}\left(\mathbf{H}_{n}\right)$ and $E \subseteq$ $\mathbf{R}^{\times}\left(\leftrightarrow \hat{G}_{r}\right)$ with Lebesgue measure $m(E)<\infty$,

$$
\left(\int_{E} \operatorname{Tr}\left(\pi_{\lambda}(f)^{*} \pi_{\lambda}(f)\right) d \mu(\lambda)\right)^{1 / 2} \leq\left(2 \theta^{2}\right)^{-\theta}(1-2 \theta)^{\theta-1} m(E)^{\theta}\left\||\cdot|_{3}^{\theta} f\right\|_{2},
$$

where $|\cdot|_{3}$ is the function $(p, q, t) \rightarrow|t|$ on $\mathbf{H}_{n}$.

5.2 REMARK. Notice that it is the concentration of $f$ in the $t$-variable which forces the nonlocalization of its (group theoretic) Fourier transform. It would be interesting to know that this is the best possible. For example, does there exist a nonzero function $f$ on $\mathbf{H}_{1}$ for which $\{(p, q, t): f(p, q, t) \neq 0\} \subseteq[-a, a] \times[-b, b] \times \mathbf{R}$ and $\left\{\lambda \in \mathbf{R}^{\times}, \pi_{\lambda}(f) \neq 0\right\} \subseteq[-c, c]$ for some $a, b, c>d$ ?

The euclidean Fourier transform of $f$ will be denoted by $F f$. Also $F_{j} f$ will denote the euclidean Fourier transform in the $j$ th (family of) variables and $F_{j k}$ the Fourier transform in the $j$ th and $k$ th (families of) variables. 
PROOF OF 5.1. It suffices to prove the result for $f \in C_{c}^{\infty}\left(\mathbf{H}_{n}\right)$. Given $\theta$ and $E$ as in the statement of the theorem, the discussion in the first part of this section gives

$$
\begin{aligned}
\int_{E} \operatorname{Tr}\left(\pi_{\lambda}(f)^{*} \pi_{\lambda}(f)\right) d \mu(\lambda) & =\int_{E} \operatorname{Tr}\left(\pi_{\lambda}(f)^{*} \pi_{\lambda}(f)\right)|\lambda|^{n} d \lambda \\
& =\int\left(\iint \frac{1}{|\lambda|^{2 n}}\left|F_{23} f(u, v, \lambda)\right|^{2}|\lambda|^{n} d u d v\right)|\lambda|^{n} d \lambda \\
& =\iint\left(\int_{E}\left|F_{3} f(u, y, \lambda)\right|^{2} d \lambda\right) d u d y
\end{aligned}
$$

where the last step is just the Parseval identity in the second family of variables followed by Fubini's theorem. From Theorem 1.1 with $k=1$ this last expression is majorized by

$$
\left(2 \theta^{2}\right)^{-2 \theta}(1-2 \theta)^{2(\theta-1)} m(E)^{2 \theta} \iiint|t|^{2 \theta}|f(p, q, t)|^{2} d p d q d t
$$

giving the required inequality.

We would like to thank G. Folland from whom we learnt the representation theory of $\mathbf{H}_{n}$ as formulated in this section.

\section{BIBLIOGRAPHY}

1. M. Benedicks, On Fourier transforms of functions supported on sets of finite Lebesgue measure, J. Math. Anal. Appl. 106 (1985), 180-183.

2. M. G. Cowling and J. F. Price, Bandwidth versus time concentration: The Heisenberg-PauliWeyl inequality, SIAM J. Math. Anal. 15 (1984), 151-165.

3. W. G. Faris, Inequalities and uncertainty principles, J. Math. Phys. 19 (1978), 461-466.

4. S. A. Gaal, Linear analysis and representation theory, Springer-Verlag, Berlin and New York, 1973.

5. R. Gangolli, Spherical functions on semisimple Lie groups, Symmetric Spaces (W. Boothby and G. Weiss, eds.), Dekker, New York, 1972, pp. 41 92.

6. S. Helgason, Differential geometry, Lie groups and symmetric spaces, Academic Press, New York, 1978.

7. I. I. Hirschman, Jr., A note on entropy, Amer. J. Math. 79 (1957), 152-156.

8. J. Ludwig, On the Hilbert-Schmidt semi-norms of $L^{2}$ of a nilpotent Lie group, Math. Ann. 273 (1986), 383-395.

9. J. F. Price, Inequalities and local uncertainty principles, J. Math. Phys. 24 (1983), 1711-1714.

10. __ Sharp local uncertainty principles, Studia Math. 85 (1987), 37-45.

11. J. F. Price and A. Sitaram, Functions and their Fourier transforms with supports of finite measure for certain locally compact groups, J. Funct. Anal. (to appear).

12. W. Schempp, Radar ambiguity functions, the Heisenberg group, and holomorphic theta series, Proc. Amer. Math. Soc. 92 (1984), 103-110.

13. M. Vergne, Representations of Lie groups and the orbit method, Emmy Noether in Bryn Mawr (B. Srinivasan and J. D. Sally, eds.), Springer-Verlag, Berlin and New York, 1983, pp. 59-101.

14. G. Warner, Harmonic analysis on semisimple Lie groups, vols. I, II, Springer-Verlag, Berlin and New York, 1972.

School of Mathematics, University of New South Wales, Kensington, NSW 2033 Australia

Statistics-Mathematics Unit, Indian Statistical Institute, Bangalore 560059 , INDIA 